\title{
Real Hypersurfaces in Complex Projective Space Whose Structure Jacobi Operator Is Cyclic-Ryan Parallel
}

JuAN DE DiOs PEREZ*

Departamento de Geometria y Topologia, Universidad de Granada, 18071 Granada, Spain

e-mail : jdperez@ugr.es

Florentino Garcia Santos

Departamento de Geometria y Topologia, Universidad de Granada, 18071 Granada, Spain

e-mail: florenti@ugr.es

ABstract. We classify real hypersurfaces in complex projective space whose structure Jacobi operator satisfies a certain cyclic condition.

\section{Introduction}

Let $\mathbb{C} P^{m}, m \geq 3$, be a complex projective space endowed with the metric $g$ of constant holomorphic sectional curvature 4 . Let $M$ be a connected real hypersurface of $\mathbb{C} P^{m}$ without boundary. Let $J$ denote the complex structure of $\mathbb{C} P^{m}$ and $N$ a locally defined unit normal vector field on $M$. Then $-J N=\xi$ is a tangent vector field to $M$ called the structure vector field on $M$. We also call $\mathbb{D}$ the maximal holomorphic distribution on $M$, that is, the distribution on $M$ given by all vectors orthogonal to $\xi$ at any point of $M$.

The study of real hypersurfaces in nonflat complex space forms is a classical topic in Differential Geometry. The classification of homogeneous real hypersurfaces in $\mathbb{C} P^{m}$ was obtained by Takagi, see [14], [15], [16], and is given by the following list: $A_{1}$ : Geodesic hyperspheres. $A_{2}$ : Tubes over totally geodesic complex projective spaces. $B$ : Tubes over complex quadrics and $\mathbb{R} P^{m} . C$ : Tubes over the Segre embedding of $\mathbb{C} P^{1} \mathrm{x} \mathbb{C} P^{n}$, where $2 n+1=m$ and $m \geq 5$. D : Tubes over the Plucker embedding of the complex Grassmann manifold $G(2,5)$. In this case $m=$ 9. $E$ : Tubes over the cannonical embedding of the Hermitian symmetric space $S O(10) / U(5)$. In this case $m=15$.

Other examples of real hypersurfaces are ruled real ones, that were introduced by Kimura, [5]: Take a regular curve $\gamma$ in $\mathbb{C} P^{m}$ with tangent vector field $X$. At each

* Corresponding author.

Received 20 June 2007; accepted 30 September 2007.

2000 Mathematics Subject Classification: 53C15, 53B25.

Key words and phrases: complex projective space, real hypersurface, structure Jacobi operator, cyclic Ryan parallelness. 
point of $\gamma$ there is a unique complex projective hyperplane cutting $\gamma$ so as to be orthogonal not only to $X$ but also to $J X$. The union of these hyperplanes is called a ruled real hypersurface. It will be an embedded hypersurface locally although globally it will in general have self-intersections and singularities. Equivalently a ruled real hypersurface is such that $\mathbb{D}$ is integrable or $g(A \mathbb{D}, \mathbb{D})=0$, where $A$ denotes the shape operator of the immersion. For further examples of ruled real hypersurfaces see [7].

Except these real hypersurfaces there are very few examples of real hypersurfaces in $\mathbb{C} P^{n}$.

On the other hand, Jacobi fields along geodesics of a given Riemannian manifold $(\tilde{M}, \tilde{g})$ satisfy a very well-known differential equation. This classical differential equation naturally inspires the so-called Jacobi operator. That is, if $\tilde{R}$ is the curvature operator of $\tilde{M}$, and $X$ is any tangent vector field to $\tilde{M}$, the Jacobi operator (with respect to $X$ ) at $p \in M, \tilde{R}_{X} \in \operatorname{End}\left(T_{p} \tilde{M}\right)$, is defined as $\left(\tilde{R}_{X} Y\right)(p)=(\tilde{R}(Y, X) X)(p)$ for all $Y \in T_{p} \tilde{M}$, being a selfadjoint endomorphism of the tangent bundle $T \tilde{M}$ of $\tilde{M}$. Clearly, each tangent vector field $X$ to $\tilde{M}$ provides a Jacobi operator with respect to $X$.

The study of Riemannian manifolds by means of their Jacobi operators has been developed following several ideas. For instance, in [1], it is pointed out that (locally) symmetric spaces of rank 1 (among them complex space forms) satisfy that all the eigenvalues of $\tilde{R}_{X}$ have constant multiplicities and are independent of the point and the tangent vector $X$.

Let $M$ be a real hypersurface in a complex projective space and let $\xi$ be the structure vector field on $M$. We will call the Jacobi operator on $M$ with respect to $\xi$ the structure Jacobi operator on $M$. Then the structure Jacobi operator $R_{\xi} \in \operatorname{End}\left(T_{p} M\right)$ is given by $\left(R_{\xi}(Y)\right)(p)=(R(Y, \xi) \xi)(p)$ for any $Y \in T_{p} M, p \in M$, where $R$ denotes the curvature operator of $M$ in $\mathbb{C} P^{m}$. Some papers devoted to study several conditions on the structure Jacobi operator of a real hypersurface in $\mathbb{C} P^{m}$ are [2], [3], [4].

Recently, [9], we have proved the non-existence of real hypersurfaces in $\mathbb{C} P^{m}$ with parallel structure Jacobi operator. Also in [10], [11], [12], [13] we have studied distinct conditions on the structure Jacobi operator (Lie parallelism, Lie $\xi$ parallelism, $\mathbb{D}$-parallelism, and so on).

For any vector fields $X, Y$ tangent to $M, R(X, Y)$ operates as a derivation on the algebra of tensor fields on $M$. For a tensor field $F$ of type $(r, s), R(X, Y) . F=$ $\nabla_{X} \nabla_{Y} F-\nabla_{Y} \nabla_{X} F-\nabla_{[X, Y]} F$. In the case of $F=R_{\xi}$, we get $\left(R(X, Y) \cdot R_{\xi}\right) Z=$ $R(X, Y)\left(R_{\xi}(Z)\right)-R_{\xi}(R(X, Y) Z)$, for any $X, Y, Z$ tangent to $M$.

The purpose of the present paper is to study a weaker condition than structure Jacobi operator being parallel for a real hypersurface of $\mathbb{C} P^{m}$. In fact we will study the condition

$$
\left(R(X, Y) \cdot R_{\xi}\right) Z+\left(R(Y, Z) \cdot R_{\xi}\right) X+\left(R(Z, X) \cdot R_{\xi}\right) Y=0
$$

for any $X, Y, Z$ tangent to $M$. Due to the literature we propose to call them real hypersurfaces with cyclic-Ryan parallel structure Jacobi operator. 
We will obtain the following

Theorem. Let $M$ be a real hypersurface of $\mathbb{C} P^{m}, m \geq 3$. Then $M$ has cyclic-Ryan parallel structure Jacobi operator if and only if $M$ is locally congruent either to a geodesic hypersphere or to a tube of radius $\pi / 4$ over a complex submanifold in $\mathbb{C} P^{m}$.

\section{Preliminaries}

Thoughout this paper, all manifolds, vector fields, etc., will be considered of class $C^{\infty}$ unless otherwise stated. Let $M$ be a connected real hypersurface in $\mathbb{C} P^{m}$, $m \geq 2$, without boundary. Let $N$ be a locally defined unit normal vector field on $M$. Let $\nabla$ be the Levi-Civita connection on $M$ and $(J, g)$ the Kaehlerian structure of $\mathbb{C} P^{m}$.

For any vector field $X$ tangent to $M$ we write $J X=\phi X+\eta(X) N$, and $-J N=\xi$. Then $(\phi, \xi, \eta, g)$ is an almost contact metric structure on $M$. That is, we have

$$
\phi^{2} X=-X+\eta(X) \xi, \quad \eta(\xi)=1, \quad g(\phi X, \phi Y)=g(X, Y)-\eta(X) \eta(Y)
$$

for any tangent vectors $X, Y$ to $M$. From (2.1) we obtain

$$
\phi \xi=0, \quad \eta(X)=g(X, \xi) .
$$

From the parallelism of $J$ we get

$$
\left(\nabla_{X} \phi\right) Y=\eta(Y) A X-g(A X, Y) \xi
$$

and

$$
\nabla_{X} \xi=\phi A X
$$

for any $X, Y$ tangent to $M$, where $A$ denotes the shape operator of the immersion. As the ambient space has holomorphic sectional curvature 4, the equations of Gauss and Codazzi are given, respectively, by

$$
\begin{aligned}
R(X, Y) Z= & g(Y, Z) X-g(X, Z) Y+g(\phi Y, Z) \phi X-g(\phi X, Z) \phi Y \\
& -2 g(\phi X, Y) \phi Z+g(A Y, Z) A X-g(A X, Z) A Y
\end{aligned}
$$

and

$$
\left(\nabla_{X} A\right) Y-\left(\nabla_{Y} A\right) X=\eta(X) \phi Y-\eta(Y) \phi X-2 g(\phi X, Y) \xi
$$

for any tangent vectors $X, Y, Z$ to $M$, where $R$ is the curvature tensor of $M$.

In the sequel we need the following results:

Theorem 2.1 ([6]). A real hypersurface $M$ of $\mathbb{C} P^{m}, m \geq 3$ satisfies $R(X, Y) A Z+$ $R(Y, Z) A X+R(Z, X) A Y=0$, for any $X, Y, Z$ tangent to $M$ if and only if it is 
locally congruent to a geodesic hypersphere.

Theorem 2.1 ([9]. There exist no real hypersurfaces $M$ in $\mathbb{C} P^{m}, m \geq 3$, such that the shape operator is given by $A \xi=\xi+\beta U, A U=\beta \xi+\left(\beta^{2}-1\right) U, A \phi U=-\phi U$, $A X=-X$, for any tangent vector $X$ orthogonal to $\operatorname{Span}\{\xi, U, \phi U\}$, where $U$ is a unit vector field in $\mathbb{D}$ and $\beta$ is a nonvanishig smooth function defined on $M$.

\section{Proof of the theorem}

Bearing in mind Bianchi identity, (1.1) is equivalent to have $R(X, Y)\left(R_{\xi}(Z)\right)+$ $R(Y, Z)\left(R_{\xi}(X)\right)+R(Z, X)\left(R_{\xi}(Y)\right)=0$. As $R_{\xi}(Z)=Z-g(Z, \xi) \xi+g(A \xi, \xi) A Z-$ $g(A Z, \xi) A \xi$, we get $R(X, Y)\left(R_{\xi}(Z)\right)=R(X, Y) Z-g(Z, \xi) R(X, Y) \xi+g(A \xi, \xi)$ $R(X, Y) A Z-g(A Z, \xi) R(X, Y) A \xi$. So our condition is equivalent to $-g(Z, \xi)$ $R(X, Y) \xi-g(X, \xi) R(Y, Z) \xi-g(Y, \xi) R(Z, X) \xi+g(A \xi, \xi)[R(X, Y) A Z+R(Y, Z) A X+$ $R(Z, X) A Y]-g(A Z, \xi) R(X, Y) A \xi-g(A Y, \xi) R(Z, X) A \xi-g(A X, \xi) R(Y, Z) A \xi=0$. From Gauss equation we obtain

$$
\begin{aligned}
(3.1) & -g(Z, \xi)(g(A Y, \xi) A X-g(A X, \xi) A Y)-g(X, \xi)(g(A Z, \xi) A Y-g(A Y, \xi) A Z) \\
& -g(Y, \xi)(g(A X, \xi) A Z-g(A Z, \xi) A X)+g(A \xi, \xi)(g(\phi Y, A Z) \phi X \\
& -g(\phi X, A Z) \phi Y-2 g(\phi X, Y) \phi A Z+g(\phi Z, A X) \phi Y-g(\phi Y, A X) \phi Z \\
& -2 g(\phi Y, Z) \phi A X+g(\phi X, A Y) \phi Z-g(\phi Z, A Y) \phi X-2 g(\phi Z, X) \phi A Y) \\
& -g(A Z, \xi)(g(\phi Y, A \xi) \phi X-g(\phi X, A \xi) \phi Y-2 g(\phi X, Y) \phi A \xi \\
& +g(A Y, A \xi) A X-g(A X, A \xi) A Y)-g(A X, \xi)(g(\phi Z, A \xi) \phi Y-g(\phi Y, A \xi) \phi Z \\
& -2 g(\phi Y, Z) \phi A \xi+g(A Z, A \xi) A Y-g(A Y, A \xi) A Z)-g(A Y, \xi)(g(\phi X, A \xi) \phi Z \\
& -g(\phi Z, A \xi) \phi X-2 g(\phi Z, X) \phi A \xi+g(A X, A \xi) A Z-g(A Z, A \xi) A X)=0
\end{aligned}
$$

for any $X, Y, Z$ tangent to $M$. First we suppose that $M$ is Hopf, that is, $A \xi=\alpha \xi$, for a certain function $\alpha$. Then (3.1) becomes

$$
\alpha(R(X, Y) A Z+R(Y, Z) A X+R(Z, X) A Y)=0
$$

for any $X, Y, Z$ tangent to $M$. Thus if $\alpha \neq 0, R(X, Y) A Z+R(Y, Z) A X+$ $R(Z, X) A Y=0$. From Theorem 2.1, $M$ must be locally congruent to a geodesic hypersphere. If $\alpha=0$, then $M$ is locally congruent to a tube of radius $\pi / 4$ over a complex submanifold of $\mathbb{C} P^{m}$.

From now on we suppose that $M$ is not Hopf. Thus locally we can write $A \xi=\alpha \xi+\beta U$, where $U$ is a unit vector field in $\mathbb{D}$ and $\beta$ a nonnull function. Introducing this expression into (3.1) we get

$$
\begin{aligned}
& -\beta g(Z, \xi)(g(Y, U) A X-g(X, U) A Y)-\beta g(X, \xi)(g(Z, U) A Y-g(Y, U) A Z) \\
& -\beta g(Y, \xi)(g(X, U) A Z-g(Z, U) A X)+\alpha(g((A \phi+\phi A) Y, Z) \phi X \\
& -2 g(\phi Y, Z) \phi A X+g((A \phi+\phi A) Z, X) \phi Y-2 g(\phi Z, X) \phi A Y
\end{aligned}
$$




$$
\begin{aligned}
& +g((A \phi+\phi A) X, Y) \phi Z-2 g(\phi X, Y) \phi A Z)-g(A Z, \xi) \\
& (\beta g(\phi Y, U) \phi X-\beta g(\phi X, U) \phi Y-2 \beta g(\phi X, Y) \phi U \\
& +g(A Y, A \xi) A X-g(A X, A \xi) A Y)-g(A X, \xi)(\beta g(\phi Z, U) \phi Y \\
& -\beta g(\phi Y, U) \phi Z-2 \beta g(\phi Y, Z) \phi U+g(A Z, A \xi) A Y \\
& -g(A Y, A \xi) A Z)-g(A Y, \xi)(\beta g(\phi X, U) \phi Z-\beta g(\phi Z, U) \phi X \\
& -2 \beta g(\phi Z, X) \phi U+g(A X, A \xi) A Z-g(A Z, A \xi) A X)=0
\end{aligned}
$$

for any $X, Y, Z$ tangent to $M$. From now on we will call $\mathbb{D}_{U}$ the subspace of $T M$ orthogonal to the subspace spanned by $\xi, U, \phi U$. Taking $Z=\xi, Y=U, X=\phi U$ in (3.3) we obtain $\beta g(A \phi U, U)=0$. Thus

$$
g(A U, \phi U)=0 .
$$

Taking $Z=\xi, Y=U, X \in \mathbb{D}_{U}$ in (3.3) we have

$$
g(A U, X)=0
$$

for any $X \in \mathbb{D}_{U}$. From(3.4) and (3.5) we obtain $A U=\beta \xi+g(A U, U) U$. If we take $Z=U, Y=\phi U, X \in \mathbb{D}_{U}$ in (3.3) we get $-\alpha g(A U, U) \phi X-\alpha g(A \phi U, \phi U) \phi X+$ $2 \alpha \phi A X-\alpha g(A \phi U, X) U+\alpha g(A \phi X, \phi U) \phi U+\beta^{2} \phi X=0$. If $\alpha=0$ this yields $\beta^{2} \phi X=0$ which is impossible. Thus $\alpha \neq 0$. Taking the scalar product with $\phi U$,

$$
g(A \phi X, \phi U)=0
$$

for any $X \in \mathbb{D}_{U}$. Thus $\phi U$ is principal and the above expression reduces to $-\alpha g(A U, U) \phi X-\alpha g(A \phi U, \phi U) \phi X+2 \alpha \phi A X+\beta^{2} \phi X=0$, for any $X \in \mathbb{D}_{U}$. If we apply $\phi$ we obtain $\alpha g(A U, U) X+\alpha g(A \phi U, \phi U) X-2 \alpha A X-\beta^{2} X=0$ for any $X \in \mathbb{D}_{U}$. It follows

$$
\left.A X=((g(A U, U)+g(A \phi U, \phi U)) / 2)-\left(\beta^{2} / 2 \alpha\right)\right) X
$$

for any $X \in \mathbb{D}_{U}$. If we take $X \in \mathbb{D}_{U}, Y=\phi X, Z=U$ in (3.3) and its scalar product with $\phi U$ we get

$$
\alpha(g(A \phi X, \phi X)+g(A X, X)-2 g(A U, U))+2 \beta^{2}=0
$$

for any $X \in \mathbb{D}_{U}$. From (3.7) and (3.8) we obtain

$$
g(A U, U)=g(A \phi U, \phi U)+\left(\beta^{2} / \alpha\right) .
$$

Taking $X \in \mathbb{D}_{U}, Y=\phi X, Z=\phi U$ in (3.3) and its scalar product with $U$ it follows

$$
\begin{gathered}
g(A \phi U, \phi U)=g(A X, X), \\
g(A U, U)=g(A X, X)+\left(\beta^{2} / \alpha\right)
\end{gathered}
$$


for any $X \in \mathbb{D}_{U}$. If we call $A \phi U=\gamma \phi U$, then $g(A U, U)=\gamma+\left(\beta^{2} / \alpha\right)$.

Consider two orthonormal vector fields $X, Y \in \mathbb{D}_{U}$. Codazzi equation gives $\left(\nabla_{X} A\right) Y-\left(\nabla_{Y} A\right) X=-2 g(\phi X, Y) \xi$. That is, $X(\gamma) Y-Y(\gamma) X+\gamma[X, Y]-$ $A[X, Y]=-2 g(\phi X, Y) \xi$. Taking the scalar product of this expression and $\xi$ we get

$$
(\gamma-\alpha) g([X, Y], \xi)-\beta g([X, Y], U)=-2 g(\phi X, Y) .
$$

And its scalar product with $U$ gives

$$
\alpha g([X, Y], \xi)+\beta g([X, Y], U)=0 .
$$

As $g([X, Y], \xi)=g\left(X, \nabla_{Y} \xi\right)-g\left(Y, \nabla_{X} \xi\right)=g(X, \phi A Y)-g(Y, \phi A X)=-2 \gamma g(\phi X, Y)$, from (3.11) and (3.12) we have

$$
\gamma^{2}=1
$$

Now if we take $X \in \mathbb{D}_{U}, Y=U, Z=\xi$ in (3.3) we obtain $(1+\gamma \alpha) \beta \gamma X=0$. This yields

$$
1+\alpha \gamma=0
$$

From (3.13) and (3.14) we have two possibilities: i) $\gamma=-1, \alpha=1$ or ii) $\gamma=1$, $\alpha=-1$.

From Theorem 2.2 case i) cannot occur. So we consider case ii), that is, $A \xi=$ $-\xi+\beta U, A U=\beta \xi+\left(1-\beta^{2}\right) U, A \phi U=U, A X=X$, for any $X \in \mathbb{D}_{U}$. Take $X \in \mathbb{D}_{U}$. Codazzi equation gives $\left(\nabla_{X} A\right) U-\left(\nabla_{U} A\right) X=0$. This yields $X(\beta) \xi+$ $\beta \phi X+X\left(1-\beta^{2}\right) U+\left(1-\beta^{2}\right) \nabla_{X} U-A \nabla_{X} U-\nabla_{U} X+A \nabla_{U} X=0$. Taking the scalar product of this equality and $U$ we get

$$
g\left(\nabla_{U} U, X\right)=2 X(\beta) / \beta,
$$

and the scalar product with $\xi$ yields

$$
g\left(\nabla_{U} U, X\right)=X(\beta) / \beta .
$$

From (3.15) and (3.16) we get

$$
X(\beta)=0
$$

for any $X \in \mathbb{D}_{U}$. The scalar product of the above expression and $X$ gives

$$
g\left(\nabla_{X} U, X\right)=0
$$

for any $X \in \mathbb{D}_{U}$.

If we develop $\left(\nabla_{X+U} A\right) \xi-\left(\nabla_{\xi} A\right)(X+U)=-\phi X-\phi U$ and take its scalar product with $X \in \mathbb{D}_{U}$ we obtain $\beta g\left(\nabla_{X} U, X\right)+\beta g\left(\nabla_{U} U, X\right)+\beta^{2} g\left(\nabla_{\xi} U, X\right)=0$. From (3.17) and (3.18) this yields

$$
g\left(\nabla_{\xi} U, X\right)=0
$$


for any $X \in \mathbb{D}_{U}$.

Developing $\left(\nabla_{X+\phi U} A\right) \xi-\left(\nabla_{\xi} A\right)(X+\phi U)=-\phi X+U$ and taking its scalar product with $U$, bearing in mind (3.17), (3.18) and (3.19) we have

$$
(\phi U)(\beta)+\left(1-2 \beta^{2}\right)-\beta^{2} g\left(\nabla_{\xi} \phi U, U\right)=0 .
$$

and taking its scalar product with $\xi$ it follows

$$
g\left(\nabla_{\xi} \phi U, U\right)=-4 .
$$

From (3.20) and (3.21) we get

$$
(\phi U)(\beta)=-\left(2 \beta^{2}+1\right) .
$$

If we develop $\left(\nabla_{U} A\right) \xi-\left(\nabla_{\xi} A\right) U=-\phi U$ and take its scalar product with $U$ we obtain

$$
U(\beta)=-2 \beta \xi(\beta)
$$

and its scalar product with $\xi$ gives

$$
\xi(\beta)=0 .
$$

From (3.17), (3.22), (3.23) and (3.24) we get

$$
\operatorname{grad}(\beta)=-\left(2 \beta^{2}+1\right) \phi U .
$$

Thus $\nabla_{X} \operatorname{grad}(\beta)=-4 \beta X(\beta) \phi U-\left(2 \beta^{2}+1\right) \nabla_{X} \phi U$ for any $X$ tangent to $M$. Therefore, for any $Y$ tangent to $M$ we have $g\left(\nabla_{X} \operatorname{grad}(\beta), Y\right)=-4 \beta X(\beta) g(\phi U, Y)-$ $\left(2 \beta^{2}+1\right) g\left(\nabla_{X} \phi U, Y\right)$. Thus $g\left(\nabla_{X} \operatorname{grad}(\beta), Y\right)-g\left(\nabla_{Y} \operatorname{grad}(\beta), X\right)=4 \beta(Y(\beta)$ $g(\phi U, X)-X(\beta) g(\phi U, Y))+\left(2 \beta^{2}+1\right)\left(g\left(\nabla_{Y} \phi U, X\right)-g\left(\nabla_{X} \phi U, Y\right)\right)$.

As $g\left(\nabla_{X} \operatorname{grad}(\beta), Y\right)-g\left(\nabla_{Y} \operatorname{grad}(\beta), X\right)=0$, it follows

$$
\begin{aligned}
& 4 \beta(Y(\beta) g(\phi U, X)-X(\beta) g(\phi U, Y)) \\
& +\left(2 \beta^{2}+1\right)\left(g\left(\nabla_{Y} \phi U, X\right)-g\left(\nabla_{X} \phi U, Y\right)\right)=0
\end{aligned}
$$

for any $X, Y$ tangent to $M$. Taking $Y=\xi$ in (3.26), for any $X$ tangent to $M$ we get $g\left(\nabla_{\xi} \phi U, X\right)=g\left(\nabla_{X} \phi U, \xi\right)$. Taking $X=U$ we get

$$
g\left(\nabla_{\xi} \phi U, U\right)=\beta^{2}-1 .
$$

From (3.21) and (3.27) we obtain $\beta^{2}=-3$, which is impossible, finishing the proof. As a consequence we obtain

Corollary 3.1. There exist no real hypersurfaces in $\mathbb{C} P^{m}, m \geq 3$ satisfying $R . R_{\xi}=$ 0.

Proof. As this condition implies that $M$ has cyclic-Ryan parallel structure Jacobi 
operator, it must be Hopf. So $A \xi=\alpha \xi$. Then if we develop $R_{\xi}(R(X, \xi) \xi)=0$, with $X \in \mathbb{D}$ such that $A X=\lambda X$, we get

$$
\alpha^{2} \lambda^{2}+2 \alpha \lambda+1=0 .
$$

If $\alpha=0,(3.28)$ gives a contradiction. Thus $M$ must be locally congruent to a geodesic hypersphere. In this case, $\alpha=2 \cot (2 r), \lambda=\cot (r), r \neq \pi / 4,0<r<\pi / 2$. Thus (3.28) does not hold and we finish the proof.

Acknowledgment. First author is partially supported by Mec-FEDER Grant MTM 2007-60731.

\section{References}

[1] Q. S. Chi, A curvature characterization of certain locally rank-one symmetric spaces, J. Diff. Geom., 28(1988), 187-202.

[2] J. T. Cho and U-H. Ki, Jacobi operators on real hypersurfaces of a complex projective space, Tsukuba J. Math., 22(1998), 145-156.

[3] J. T. Cho and U-H. Ki, Real hypersurfaces of a complex projective space in terms of the Jacobi operators, Acta Math. Hungar., 80(1998), 155-167.

[4] U-H. Ki, H.J. Kim and A.A. Lee, The Jacobi operator of real hypersurfaces in a complex Space form, Commun. Korean Math. Soc., 13(1998), 545-600.

[5] M. Kimura, Sectional curvatures of holomorphic planes on a real hypersurface in $P^{n}(\mathbb{C})$, Math. Ann., 276(1987), 487-497.

[6] M. Kimura and S. Maeda, On real hypersurfaces of a complex projective space III, Hokkaido Math. J., 22(1993), 63-78.

[7] M. Loknherr and H. Reckziegel, On ruled real hypersurfaces in complex space forms, Geom. Dedicata, 74(1999), 267-286.

[8] M. Okumura, On some real hypersurfaces of a c omplex projective space, Trans. A. M. S., 212(1975), 355-364.

[9] M. Ortega, J. D. Perez and F. G. Santos, Non-existence of real hypersurfaces with parallel structure Jacobi operator in nonflat complex space forms, Rocky Mountain J. Math., 36(2006), 1603-1613.

[10] J. D. Perez and F. G. Santos, On the Lie derivative of structure Jacobi operator of real hypersurfaces in complex projective space, Publ. Math. Debrecen, 66(2005), 269-282.

[11] J. D. Perez and F. G. Santos, Real hypersurfaces in complex projective space with recurrent structure Jacobi operator, Diff. Geom. Appl., 26(2008), 218-223.

[12] J. D. Perez, F. G. Santos and Y. J. Suh, Real hypersurfaces in complex projective space whose structure Jacobi operator is Lie $\xi$-parallel, Diff. Geom. Appl., 22(2005),181-188.

[13] J. D. Perez, F. G. Santos and Y. J. Suh, Real hypersurfaces in complex projective space whose structure Jacobi operator is $\mathbb{D}$-parallel, Bull. Belgian Math. Soc. Simon Stevin, 13(2006), 459-469. 
[14] R. Takagi, On homogeneous real hypersurfaces in a complex projective space, Osaka J. Math., 10(1973), 495-506.

[15] R. Takagi, Real hypersurfaces in complex projective space with constant principal curvatures, J. Math. Soc. Japan, 27(1975), 43-53.

[16] R. Takagi, Real hypersurfaces in complex projective space with constant principal curvatures II, J. Math. Soc. Japan, 27(1975), 507-516. 